\title{
NUEVAS ESTRATEGIAS ECONÓMICAS EN LA UNIVERSIDAD DESDE LA RESPONSABILIDAD SOCIAL
}

\author{
Fernando Casani, Carmen Pérez-Esparrells, Jesús Rodríguez ${ }^{1}$
}

\begin{abstract}
RESUMEN
Con la plena implantación del Espacio Europeo de Educación Superior (EEES), el contexto de referencia de las universidades españolas ya no radica en sus fronteras o incluso en las de la UE-27 (Unión Europea -27), sino que se extiende a 48 países europeos y más de 4.000 instituciones de educación superior. Por este motivo, las universidades españolas tienen que abrir sus horizontes: formular nuevas estrategias de cara a la internacionalización y buscar fuentes de financiación innovadoras. Se trata de crear un entorno académico, científico, emprendedor y creativo para tener una mayor visibilidad internacional y una mayor repercusión en el entorno económico y social.

En este contexto, dos nuevos retos se tornan claves en el desarrollo futuro de las universidades españolas: la diversificación y búsqueda de nuevas fuentes de financiación, concretamente en la figura del fundraising o captación de fondos de aquellas personas, entidades, antiguos alumnos, empresas, organizaciones, fundaciones, etc. que quieran compartir los objetivos y misiones de la universidad y el concepto de responsabilidad social universitaria. Las instituciones de educación superior tienen que responder ante más partes interesadas y rendir cuentas en un mayor ámbito de sus actividades, no sólo docencia e investigación, sino también la llamada tercera misión. Por ello, tienen que efectuar elecciones estratégicas sobre las actividades que van a realizar y las funciones que van a cumplir para poder desarrollarlas con el nivel de calidad y exigencia adecuados.
\end{abstract}

Palabras clave: universidades españolas, financiamiento, fundraising, responsabilidad social universitaria.

\section{NEW ECONOMIC STRATEGIES IN UNIVERSITIES FROM THE SOCIAL ACCOUNTABILITY PERSPECTIVE}

\section{ABSTRACT}

With the full implementation of the European Higher Education Area (EHEA), the benchmarking context of Spanish universities is not longer within its national frontiers or the EU-27 frontiers but is extended to forty eight European countries and more than four thousand higher education institutions.

For this reason, Spanish universities should open their horizons promoting the development of new strategies to face the internationalization and seeking for new sources of financing. The idea is to develop a favorable environment (academic, scientific, entrepreneurial \& innovative) in order to get a higher international visibility as well as a higher impact on the social and economic spheres.

Therefore, Spanish universities have to deal with two key challenges: the diversification and search of new sources of financing - specifically by fundraising from people, institutions, alumni, organizations and foundations sharing the mission and strategic objectives - and the notion of social accountability of the University itself.

In this context, higher education institutions have to respond before a wider group of stakeholders, not only in teaching and research but also in the so-called "third mission" (social accountability). For this reason, they have to make strategic choices on activities and projected functions in order to obtain the intended quality level.

Key words: Spanish universities, fundraising, financing, university social accountability.

1 Universidad Autónoma de Madrid, España. Contacto: carmen.perez@uam.es 


\section{NUEVAS ESTRATEGIAS ECONÓMICAS EN LA UNIVERSIDAD DESDE LA RESPONSABILIDAD SOCIAL}

\section{Introducción}

La creación del espacio europeo de educación superior (EEES) supone la necesidad de internacionalizar y modernizar las universidades españolas para cumplir un nuevo papel en la Europa del conocimiento. Dentro del "programa de trabajo para la educación y formación 2010", la Comisión Europea ha generado distintas comunicaciones y recomendaciones (European Commission, 2003, 2005 y 2006) sobre esta necesidad de adecuación y modernización de las instituciones europeas de educación superior. Se busca, entre otras cuestiones, que la universidad tenga una actuación más destacada en la mejora de la innovación y la competitividad del sistema productivo.

En este contexto, se define como una de las nuevas señas de identidad de la universidad la relación con su entorno social y económico en lo que se viene denominando la responsabilidad social o la "tercera" misión (Bueno y Casani, 2007).

El concepto de tercera misión de la universidad puede enfocarse desde dos perspectivas: desde la de los ingresos aparece la idea de universidad emprendedora (Clark,1998) que implica una nueva fuente de ingresos a través de distintas actividades, como la formación permanente, los contratos con empresas, la comercialización de tecnología, spinoffs, merchandising, fundraising, etc. Como remarca este autor, la universidad emprendedora representa el nuevo paradigma para la búsqueda del éxito en el ámbito global y competitivo de la educación superior (Clark, 1998). Esta estrategia precisa la existencia de un núcleo fuerte de gestión y una periferia emprendedora que comparten una misma visión de la universidad.

Según Casani (2007) implica, por una parte, una especialización en aquellas actividades de enseñanza e investigación en las que tienen 
mayores fortalezas para competir en el mercado de la educación superior, y por otra, una necesidad de enfocarse en la transferencia del conocimiento generado a la sociedad, y conseguir, a través de sus actuaciones de compromiso con ésta que financie de forma más directa sus actividades. Se incluyen aquí dos maneras de conseguir ingresos: mediante la venta y explotación de sus productos de conocimiento, y a través de la captación de fondos entregados de forma voluntaria por los agentes sociales. Esta última forma de captación de fondos, que implica un fuerte reconocimiento de su aportación a la sociedad, se conoce como la función del fundraising en el mundo anglosajón.

Desde la perspectiva de los gastos se observa cómo el concepto de tercera misión y su vinculación con la comunidad en la que se inserta la universidad (community outreach) puede suponer una generación de gastos para actividades como inserción y desarrollo social, desarrollo regional y urbano, etc. Estas actividades que, a semejanza de lo que ocurre en el mundo empresarial, se podrían agrupar bajo el epígrafe de la responsabilidad social de la universidad, reflejan la aportación que la universidad realiza al entorno en el que se encuentra, además de sus funciones específicas de docencia e investigación.

Esta relación de la universidad con su entorno económico y social es fundamental para mejorar la competitividad empresarial y el desarrollo social, y afecta muy directamente a la financiación de la propia universidad que, en un momento de disminución del gasto público como el actual, tiene que recurrir a la financiación privada para su sostenimiento.

La financiación de las universidades públicas en España se basa fundamentalmente en recursos públicos. En los últimos tiempos el análisis de la financiación y la calidad de las universidades ha sido una constante en los debates propuestos por el Ministerio de Educación en España (Pérez Esparrells, 2004). De hecho, en menos de cinco años se han redactado dos Informes sobre Política de Financiación Universitaria (Comisión de Financiación, 2007 y 2010) que aconsejan la diversificación de las fuentes de financiación. Se tendrían que implementar otros mecanismos de financiación que contribuyan a optimizar los recursos disponibles por parte de las universidades, así 
como a la captación de fondos de origen privado. Las universidades históricamente han podido captar fondos privados a través de donaciones voluntarias. Este sistema tiene una amplia tradición en los Estados Unidos, sin embargo en Europa, salvo algunas contadas excepciones, no tiene prácticamente repercusión en la financiación de las universidades. A través de la gestión basada en la responsabilidad social corporativa, las instituciones de educación superior pueden aportar su esfuerzo al desarrollo económico y social de la colectividad y al mismo tiempo conseguir, a través del fundraising, fondos para su mantenimiento. En esta comunicación vamos a explorar estos dos conceptos dentro del ámbito de la misión de la universidad de apoyo a la innovación y mejora de la competitividad del sistema productivo.

\section{La responsabilidad social en las universidades}

La responsabilidad social corporativa (RSC) se entiende como el compromiso voluntario de las empresas y organizaciones con el desarrollo de la sociedad y la preservación del medioambiente, desde su composición social, y con un comportamiento responsable hacia las personas y grupos sociales con quienes se interactúa (AECA, 2006).

El enfoque de RS está relacionado con el concepto de sostenibilidad de la organización. La sostenibilidad es la capacidad de la organización para mantenerse en el tiempo porque satisface las necesidades de todas las partes interesadas con las que interactúa y conserva los recursos para su utilización futura. En este sentido, se puede afirmar que la sostenibilidad hace referencia a la capacidad de la empresa por mantenerse en el largo plazo sin descuidar los resultados a corto. Para algunos autores, la sostenibilidad consiste en resolver las necesidades del presente sin comprometer la capacidad de las generaciones futuras para resolver las suyas. Por tanto, se pone el énfasis no sólo en aspectos económicos, sino también en aquellos aspectos medioambientales y sociales.

Las empresas no son exclusivamente meras organizaciones dedicadas a crear valor para sus accionistas, sino que también tienen un compromiso con el desarrollo de la sociedad, el medioambiente, 
la cultura, la integración social o el buen gobierno. Según el Foro de Expertos en RSC del Ministerio de Trabajo, "la RS de la empresa es, además del cumplimiento estricto de las obligaciones legales vigentes, la integración voluntaria en su gobierno y gestión, en su estrategia, políticas y procedimientos, de las preocupaciones sociales, laborales, medioambientales y de respeto a los derechos humanos que surgen de la relación y el diálogo transparentes con sus grupos de interés, responsabilizándose así de las consecuencias y los impactos que se derivan de sus acciones".

En la práctica, bajo la etiqueta de responsabilidad social se hace referencia a distintos aspectos en el ámbito empresarial. Por una parte, tiene un componente ético y se centra en los valores que deben guiar la actuación de la empresa y el comportamiento de sus directivos. El objetivo último de la empresa no puede ser solo la obtención del máximo beneficio a corto plazo, sino que también tiene otros compromisos de orden social y medioambiental que deben ser tenidos en cuenta. Se desarrolla así el concepto de empresa ciudadana (Lozano, 2001).

A pesar de que la responsabilidad social se define como una opción voluntaria de las empresas, en el ámbito público existen numerosas iniciativas de institucionalizarla, ya sea a través de la creación de opinión, impulsada por organizaciones sociales y organizaciones no gubernamentales (ONGs), o mediante iniciativas políticas, encaminadas a hacer un seguimiento de las actividades de las empresas en esta área, e incluso a introducir sus prácticas en la Administración Pública (observatorios, libros blancos, comisiones parlamentarias, etc.) (Lozano, 2007).

Tradicionalmente se ha identificado la responsabilidad de la empresa con las prácticas de filantropía de los grandes empresarios, quienes a través de sus fundaciones, sobre todo en el mundo anglosajón, revierten parte de sus fortunas a determinadas causas sociales o de investigación. Dentro de la propia empresa las actividades definidas como acción social hacia su entorno o para sus propios trabajadores también suelen ser englobadas en la actualidad bajo la rúbrica de la responsabilidad social. Estas acciones representan un gasto voluntario 
de la empresa para apoyar determinados proyectos sociales o para mejorar las condiciones laborales de sus trabajadores.

Por último, la importancia que está alcanzando este concepto en la empresa en la actualidad tiene mucho que ver con el desarrollo de un modelo de gestión que, bajo esta denominación, pretende reducir riesgos y aprovechar oportunidades para mejorar la competitividad de la empresa en el nuevo entorno competitivo en el que tiene que desarrollar sus actividades. Este último aspecto, más novedoso y completo es el que va a influir sobre la forma de gestionar otras instituciones sin ánimo de lucro y, más concretamente, sobre las propias universidades.

En la tabla $n .{ }^{\circ} 1$ se recogen de forma sintética los distintos aspectos a los que puede hacer referencia el concepto de responsabilidad de la empresa. En la actualidad se está consolidando como un sistema de gestión enfocado a informar y satisfacer las necesidades de los distintos grupos de interés relacionados con la organización.

Tabla n. ${ }^{\circ}$ 1. Aspectos de la Responsabilidad Social de la Empresa (RSE).

\begin{tabular}{|l|l|l|l|l|}
\hline \multirow{2}{*}{ RSC } & \multicolumn{3}{|c|}{ Aspectos } \\
\cline { 2 - 5 } & \multicolumn{1}{|c|}{ Ético } & Políticas públicas & Acción social & \multicolumn{1}{|c|}{ Modelo de gestión } \\
\hline EMPRESA & $\begin{array}{l}\text { La empresa } \\
\text { ciudadana }\end{array}$ & $\begin{array}{l}\text { Libros blancos } \\
\text { Consejos } \\
\text { Observatorios }\end{array}$ & $\begin{array}{l}\text { Filantropía y } \\
\text { acción social }\end{array}$ & $\begin{array}{l}\text { Enfoque grupos de interés } \\
\text { Código de buen gobierno } \\
\text { Comportamientos }\end{array}$ \\
& $\begin{array}{lll}\text { Comoria sostenibilidad } \\
\text { directivos }\end{array}$ & & $\begin{array}{l}\text { Dimensión: } \\
\text { - Económica } \\
\text { - Ambiental } \\
\text { - Social }\end{array}$ \\
\hline
\end{tabular}

Fuente: elaboración propia.

Las características de la adopción de un modelo de gestión de responsabilidad social en las empresas son muy diversas y se pueden corresponder con los siguientes puntos (Casani y Pérez Esparrells, 2009):

1. La RS se entiende como un compromiso voluntario en que se define la misión, visión y valores de la empresa.

2. Es necesario identificar y reconocer a los diferentes grupos de interés, no sólo los accionistas, sino a cualquier grupo con un 
interés en la organización. Los grupos de interés se perfilan en función de su poder, legitimidad y urgencia (Mitchell, Agle, y Wood, 1997).

3. Se deben definir las políticas para cada uno de los grupos y sus temas de interés, lo que se conoce con el nombre de RS estratégica.

4. Se debe establecer un diálogo con los distintos stakeholders para garantizar que sus intereses sean considerados en los órganos de gobierno (AECA, 2007).

5. Se debe elaborar una Memoria de RSC o de sostenibilidad según las normas de un marco de referencia. En España, el estándar que más se ha utilizado es el GRI (Global Reporting Initiative, 2006).

6. Es necesario buscar indicadores para medir los resultados de la RSC.

7. Las organizaciones se preocupan por cuestiones tales como ¿cuál es el coste de la RSC?; ¿Cómo se pueden contabilizar los beneficios generados? Normalmente utilizan distintas herramientas como el análisis coste-beneficio, los índices de reputación, etc.

La RSC, como modelo de gestión, es aplicable a cualquier tipo de organización: privada o pública, grande o pequeña. Por una parte, las organizaciones privadas tienen que modificar sus sistemas de gobierno y de gestión para que permitan participar y estar informados a distintos grupos de interés (stakeholders) que persiguen distintos objetivos del tradicional valor para el accionista y que tienen que ser asumidos por la empresa para garantizar su sostenibilidad en el tiempo. Por otra, las organizaciones públicas y las entidades sin ánimo de lucro, como son las universidades públicas, tienen que incorporar a sus propios sistemas las herramientas empresariales que mejoren su eficiencia, transparencia y rendición de cuentas a la sociedad. Este enfoque de la responsabilidad social corporativa es, por tanto, especialmente adecuado para que las organizaciones públicas asuman el modelo de gestión empresarial manteniendo al mismo tiempo su propia naturaleza de rentabilidad social y no estrictamente economicista (Casani, Rodríguez Pomeda y Martín, 2007).

Por lo que respecta a las universidades, éstas tradicionalmente han perseguido unos objetivos de carácter social, por lo que dichos 
objetivos los han integrado, con mayor o menor éxito, en sus sistemas de gobierno. En los últimos años, se han introducido nuevas prácticas de gestión a través de lo que se conoce como la nueva gestión pública o el enfoque de la calidad de los servicios. La adopción de modelos de responsabilidad social representa un nuevo paso en la mejora de los sistemas de gobierno y gestión de la universidad ${ }^{2}$. Existe una demanda en todo el mundo ${ }^{3}$ de sistemas más transparentes de responsabilidad social y de rendición de cuentas para las Instituciones de Educación Superior. En todo caso, en España donde el 85\% del presupuesto de las universidades públicas se financia con fondos públicos, parece necesario que, no solo la comunidad universitaria y la administración financiadora, sino también el conjunto de la sociedad tengan un derecho legítimo a conocer mejor el modo en el que se usan los recursos públicos destinados a las instituciones universitarias y cuáles son los resultados que se obtienen.

Se trataría, entre otros muchos aspectos, de una rendición de cuentas horizontal materializada en la elaboración y presentación de una Memoria de $\mathrm{RS}^{4}$, que abarcaría también la dimensión social que existe en cualquier universidad pública.

De la misma manera que la RSC abarca distintos aspectos en el ámbito empresarial, en la universidad también puede hacer referencia a distintas cuestiones, que vamos a ordenar, en la tabla n. ${ }^{\circ}$ 2 , de la misma manera que lo hemos hecho para las empresas en la tabla n. ${ }^{\circ} 1$.

2 La importancia de la responsabilidad social y el cambio en la gestión de las universidades han sido señaladas por numerosos autores desde comienzos del siglo entre los que destacan: Neave (2000), Scott (2003) y Shattock (2003).

3 En Estados Unidos, la Administración de B. Obama considera prioritaria una nueva forma de evaluar el sistema de educación superior antes de destinar nuevos fondos estatales a este servicio educativo.

4 En algunas universidades se elaboran ya Memorias de Responsabilidad Social (como en la Universidad de Zaragoza, Santiago de Compostela, etc.), y en la actualidad, el conjunto de las universidades públicas andaluzas están elaborando una misma estructura de Memoria, auspiciadas por los Consejos Sociales de Andalucía. 
Tabla n. ${ }^{\circ}$ 2. Aspectos de la Responsabilidad Social de la Universidad (RSU).

\begin{tabular}{|c|c|c|c|c|}
\hline \multirow[t]{2}{*}{ RS } & \multicolumn{4}{|c|}{ Aspectos } \\
\hline & Ético & Políticas públicas & Acción social & Modelo de gestión \\
\hline \multirow[t]{2}{*}{ UNIVERSIDAD } & \begin{tabular}{|l|} 
Valores \\
-En la propia \\
universidad \\
$*$ Misión \\
*Visión \\
-Transmisión a \\
los estudiantes
\end{tabular} & \begin{tabular}{|l} 
Integración en \\
las políticas y \\
colaborar en su \\
desarrollo
\end{tabular} & $\begin{array}{l}\text { Cooperación y } \\
\text { acción solidaria } \\
\text { y voluntariado }\end{array}$ & $\begin{array}{l}\text { Enfoque grupos de interés } \\
\text { Código de buen gobierno } \\
\text { Memoria sostenibilidad } \\
\text { Dimensión: } \\
\text { - Económica } \\
\text { - Ambiental } \\
\text { - Social }\end{array}$ \\
\hline & $\begin{array}{l}\text { Indicadores n. }^{\circ} \\
\text { - Asignaturas } \\
\text { - Titulaciones } \\
\text { - Contenidos y } \\
\text { competencias }\end{array}$ & $\begin{array}{l}\text { Indicadores n. } \\
\text { Participaciones } \\
\text { Proyectos } \\
\text { Foros } \\
\text { Consejos }\end{array}$ & $\begin{array}{l}\text { Indicadores n. }^{\circ} \\
\text {-Voluntarios } \\
\text {-Acciones } \\
\text { cooperación }\end{array}$ & \begin{tabular}{|l|} 
Indicadores \\
-Adaptación Memoria-GRI \\
-Indicadores de gobierno \\
-Indicadores ambientales
\end{tabular} \\
\hline
\end{tabular}

Fuente: elaboración propia.

Desde la perspectiva de los valores que deben guiar la actuación de las organizaciones, la universidad tendría que asumir una doble responsabilidad. Por una parte, introducir estos valores en su propio comportamiento y, por tanto, incorporarlos a sus objetivos últimos reflejados en su misión y visión; y por otra, transmitir a sus estudiantes la importancia de considerar los valores éticos para el futuro desarrollo de sus carreras profesionales.

La participación de las universidades en las políticas públicas de impulso de la responsabilidad social de las organizaciones puede manifestarse de diferentes maneras, ya sea a partir del protagonismo de la propia institución en las distintas iniciativas o mediante la intervención de algunos de sus profesores en los distintos observatorios, foros o comisiones que se dedican a potenciar esta actividad.

La acción social de la universidad se manifiesta, además de las actuaciones específicas en esta área con su propio personal, en los programas de cooperación, acción solidaria y voluntariado que promueven la mayoría de las instituciones de educación superior en nuestro país.

Por último, la introducción del modelo de responsabilidad social en los mecanismos de gestión de la universidad todavía está poco desarrollado; aunque existen distintas iniciativas, como se ha 
señalado, algunas de ellas impulsadas por los Consejos Sociales, de avanzar en la presentación de la memoria de sostenibilidad de la institución.

La implantación de un modelo de gestión de responsabilidad social en las universidades estaría caracterizada por una serie de aspectos que, a semejanza del esquema planteado para la empresa, serían los siguientes (Casani y Pérez Esparrells, 2009):

La RSU no debería ser una opción voluntaria como en la empresa, ya que es intrínseca a la naturaleza de la institución y tradicionalmente está contenida en la misión y en los valores de la educación universitaria. Sin embargo, el incorporarla en sus sistemas de gobierno y gestión sí que sería una herramienta nueva para las universidades.

Se debe identificar el mapa de stakeholders, distinguiendo en función de su importancia y poder actual, al tiempo que se debería reflexionar si deberían tener los diferentes grupos de interés (internos y externos) más o menos poder y mayor o menor influencia.

Las decisiones estratégicas deben tener en cuenta los intereses de los distintos stakeholders (que pueden converger en algunos puntos y en otros no). Por ello, las elecciones estratégicas (mapa de titulaciones, áreas prioritarias de investigación y transferencia del conocimiento) deben recoger un compromiso entre los diferentes intereses de los grupos que integran la Universidad y los objetivos de la institución en su conjunto.

La gobernanza debe recoger las relaciones con los distintos grupos de interés por lo que se empiezan a plantear modelos en red, la llamada gobernanza reticular (networked governance) (Jongbloed, Enders y Salerno, 2008).

La rendición de cuentas deber ser horizontal (a través de la Memoria de RS) además de la tradicional vertical recogida en las obligaciones legales: las universidades actualmente tienen que rendir cuentas a la Administración financiadora, a la Cámara de Cuentas, a la IGAE, etc. 
Por último, si se implanta un modelo de gestión de responsabilidad social en la universidad se deben establecer, a semejanza de lo que se plantea en el mundo empresarial, unos indicadores de seguimiento de los resultados obtenidos por la aplicación de dicho modelo.

La ventaja de la adopción de un modelo de responsabilidad social para la universidad, además de una mejora en sus sistemas de gestión, puede estar asociada a la atracción y captación de recursos económicos de la sociedad. El enfoque stakeholder implica recoger las necesidades de cada uno de los grupos de interés y comunicarse de forma periódica con ellos. De esta manera, a través de la relación directa con los antiguos alumnos y otros agentes (empresas, instituciones privadas, fundaciones, etc.) a los que se explica la labor que realiza la universidad, se pueden poner en marcha campañas de captación de fondos y desarrollar de una forma sistemática la función del fundraising.

\section{El fundraising o la captación de fondos en las universidades}

Desde la perspectiva económica, en el desarrollo de su misión de apoyo a la innovación y al desarrollo del sistema productivo, la universidad puede fomentar un sistema de captación de fondos (fundraising) impulsado por su mayor vinculación a los agentes económicos y sociales a través de este modelo de gestión de la responsabilidad social. Los sistemas profesionales de captación de recursos que aplican las principales universidades más prestigiosas en la actualidad se refieren a un concepto más amplio, el de "estrategia institucional" (institutional advancement $t^{5}$ ), que se concreta en la llamada "función de desarrollo" (development ${ }^{6}$ ) y que hace referencia al desarrollo futuro de la institución, intentando implicar a los posibles donantes en las inversiones y el avance de la universidad.

5 Según el Task Force report (Department for Education and Skills, 2004) se trata de un método integrado y sistemático de relaciones para incrementar el apoyo a la institución de sus stakeholders. Comprende acciones de comunicación y marketing, relaciones con los antiguos alumnos y el desarrollo.

6 El término "desarrollo" es usado en el sector de la educación superior para denotar captación de fondos y construcción de relaciones con antiguos alumnos y stakeholders. 
El concepto de fundraising surge muy ligado a la filantropía y a las organizaciones sin fines de lucro. El desarrollo institucional es una actividad altamente profesionalizada en Estados Unidos, menos extendida en Europa, y prácticamente desconocida en España. Hace referencia a tres áreas planificadas y coordinadas entre sí: la relación con los antiguos alumnos, la captación de fondos, y las relaciones externas y la comunicación pública.

El fundraising es episódico, esto es, representa un mecanismo (una oportunidad) para que las universidades capten fondos de forma voluntaria y esporádica (Mora y Nugent, 1995) y no recoge una modalidad de financiación permanente. Por el contrario, la actividad del desarrollo institucional sí tiene un carácter continuo. El desarrollo institucional es un compromiso genérico y a largo plazo del crecimiento financiero y espacial de la institución, responsabilidad conjunta de la propia institución a través de sus actividades y de los poderes públicos que participan en su financiación básica.

Como se ha puesto de manifiesto en el apartado anterior, una vía nueva de financiación en España, para que las universidades cumplan su tercera misión, podría ser potenciar el fundraising (entendido como la financiación procedente de particulares y empresas) con el fin de buscar personas o entidades que quieran compartir los objetivos y resultados de una institución universitaria mediante aportaciones económicas. Esta nueva fuente de ingresos complementaría la financiación pública que ya reciben las universidades a través de los modelos de financiación universitaria de las comunidades autónomas y los fondos de investigación estatales y europeos, así como los ingresos privados procedentes de precios públicos, tasas, ventas de servicios, contratos, etc.

Actualmente, en España no está extendida la tradición de la donación a las universidades ni tampoco la de pedir financiación a los agentes privados por parte de las instituciones universitarias de carácter público. En la Ley de Mecenazgo de $2002^{7}$ se contempla a las universidades como entidades públicas beneficiarias (art. 16.

7 Ley n. ${ }^{\circ}$ 49/2002, de 23 de diciembre, de régimen fiscal de las entidades sin fines lucrativos $\mathrm{y}$ de los incentivos fiscales al mecenazgo. 
c: "las universidades públicas y los colegios mayores adscritos a las mismas"). En el título III de incentivos fiscales al mecenazgo se establece el régimen fiscal de las donaciones y aportaciones a estas entidades: por un lado, se pueden hacer aportaciones de los particulares (25\% de deducción en la cuota) o de las empresas (35\% de deducción en la cuota). Son deducciones en la cuota íntegra del Impuesto sobre la Renta de las Personas Físicas (IRPF) (en el primer caso $^{8}$ ) y en la cuota íntegra del Impuesto sobre Sociedades (IS) (en el segundo $\operatorname{caso}^{9}$ ). Además, se trata de donaciones y aportaciones que no tienen carácter finalista. Por tanto, los particulares o las empresas que realizan esas donaciones no pueden indicar o condicionar la aplicación de esos fondos a ningún objetivo concreto, por lo que será difícil que se identifiquen con los objetivos, funciones y misiones de las universidades.

Pero no basta con la introducción de cambios en la fiscalidad, las universidades tienen que apostar fuertemente por la implicación empresarial en sus actividades de investigación y desarrollo (I+D). Hay una figura poco conocida pero que se desarrolla en la Ley de Presupuestos de cada año (en su disposición transitoria) y que cada vez más se está empezando a utilizar en España para incentivar actividades prioritarias de mecenazgo. En concreto, son las aportaciones para investigación en instalaciones científicas (ejemplos del año 2010 son la central de tecnología del Instituto de Sistemas Opto-electrónicos de la Universidad Politécnica de Madrid, el Centro de Microscopía de la Universidad Complutense de Madrid, los nodos de la Red Española de Supercomputación en las Universidades de Valencia, Málaga, Cantabria, Politécnica de Madrid, etc.) que se

8 En impuestos con tarifas progresivas, como es el caso del IRPF en España, es más regresiva una reducción sobre la base que una deducción sobre la cuota. Las reducciones sobre la base imponible implican un ahorro impositivo al tipo marginal, por lo que benefician más a los individuos de mayor renta. Por contra, las deducciones de cantidades fijas sobre la cuota (que implican un ahorro al tipo medio) benefician menos, en términos relativos, a los individuos de mayor renta. Simplemente, un cambio de la deducción en cuota a base podría incentivar a un número potencial de contribuyentes de tramos altos de renta para que efectuaran este tipo de donaciones voluntarias a las universidades.

9 En Francia en la actualidad llega hasta el 60\%. En nuestra opinión, en España en el Impuesto de Sociedad se debería aumentar siempre por encima del 30\% (que supone neutralidad en el impuesto) y, al menos, al 35\%-40\%, lo que implicaría un beneficio fiscal para las empresas. 
traducen en convenios de colaboración de las empresas para la investigación en esos centros. Bajo esta fórmula, la empresa sí que influye directamente en el destino de los fondos procedentes de sus aportaciones o donaciones. Por tanto, se trata de aportaciones de carácter finalista a las universidades. No obstante, son relativamente pocas las donaciones que se canalizan bajo esta fórmula que potencia, sin duda, la imbricación universidad-empresa-universidad, ya que son las propias empresas las que indican (condicionan) la aplicación de esos fondos a un objetivo concreto.

En España, la tradición de los universitarios (antiguos alumnos) por continuar vinculados a la institución universitaria en la que recibieron su formación está muy poco desarrollada y nos encontramos muy lejos de esta perspectiva económica (y cultural) de aportación individual anual que existe, por ejemplo, en muchas universidades americanas. Tampoco existe la figura de los legados. Por ello, es necesaria una reforma de la Ley de Mecenazgo. Es un proceso largo y costoso, pero que merecería la pena dados los importantes logros que se conseguirían tras su puesta en marcha. La idiosincrasia española respecto a este tema está muy alejada de la realidad en Estados Unidos. Por tanto, puede verse como un desafío cultural para académicos, estudiosos del tema, gobernantes (en los niveles europeo, central y autonómico), y partidos políticos en el sentido de un cambio en su conducta y discurso. Tal cambio iría encaminado hacia una adecuada explicación a la sociedad de las ventajas que se derivan del fundraising, para promoverlo y orientar sus respectivas políticas en esa dirección (Porter y Kramer, 2006).

En nuestra opinión, para potenciar la captación de fondos por las universidades españolas es preciso, en primer lugar, un diagnóstico correcto que parta de la situación actual de las universidades públicas españolas. En ellas las actividades relacionadas con la transferencia del conocimiento representan unas cantidades relativamente muy pequeñas con respecto al total de los presupuestos (alrededor del 5\%) y no todas provienen de fondos privados. Respecto a las donaciones (o fundraising propiamente dicho), son cantidades insignificantes que no se pueden detectar fácilmente en los estados contables. Por lo tanto, para conseguir un cambio cuantitativo (mayores donaciones) 
el cambio debe ser cualitativo. Para impulsarlo habría que realizar acciones que fomentasen, por una parte, la "cultura de dar" de la sociedad, y por otra, "la cultura de pedir" de las universidades.

En cuanto a cómo cambiar la cultura del "dar" (culture of giving) se pueden ir implantando una serie de medidas como:

- Introducir nuevos incentivos fiscales (Ley de Ciencia)

- Incentivar cambios en el espíritu filantrópico en la sociedad española

- Potenciar la diferenciación de las universidades (caso alemán: universidades altamente investigadoras, universidades especializadas en FP) en su imagen de marca y en su posicionamiento estratégico (con el entorno socioeconómico europeo, nacional, regional y local) (European Commission, 2008)

- Fomentar la identificación de los estudiantes con la universidad (Kaplan, 2009).

Al mismo tiempo y más importante, es necesario cambiar la cultura del "pedir" (culture of asking). Para ello, será necesario ir introduciendo en las universidades españolas una serie de medidas como:

- Creación de una Oficina de Desarrollo Institucional (Development Office) (Gilby, Lloyd y Finch, 2009)

- Cuidar y potenciar la atención a los antiguos alumnos

- Crear un clima de confianza académica hacia el mundo empresarial

- Impulsar los lazos con las empresas que participan activamente con la universidad en la Formación Continua.

\section{Conclusiones}

En los últimos años se está produciendo en todos los países un cambio en la percepción del lugar que tiene que ocupar la universidad en la sociedad, dentro de un ámbito cada vez más internacional. El compromiso social creciente de la universidad con la obtención de resultados para el entorno se concreta en una atención preferente hacia las demandas cambiantes de una sociedad cada vez más integrada en 
un mundo abierto y dinámico, sin abandonar por ello la necesidad de preservar el núcleo del valor de la institución universitaria: la docencia y la investigación de excelencia y calidad. En concreto, existe un consenso que destaca la importancia de la universidad como catalizador y motor del desarrollo económico y social, especialmente en un momento de crisis económica como el actual, en el que existe la necesidad de adoptar reformas para equilibrar el peso de los diferentes sectores.

Para realizar esta función, integrada dentro del concepto amplio de tercera misión, la universidad necesita una financiación estable que le garantice su sostenibilidad en el tiempo. El debate sobre la financiación de la universidad se está decantando hacia complementar los fondos públicos con aportaciones privadas ligadas a su propia actividad. La implantación de herramientas de responsabilidad social y de fundraising va a permitir a las instituciones de educación superior acceder con mayor facilidad a los fondos privados y mejorar su visibilidad.

Como se ha remarcado, el modelo de RS que está resultando válido para el mundo de la empresa puede resultar igualmente válido para el mundo de la universidad, ya que un modelo de gestión que ponga el énfasis en aspectos económicos, medioambientales y sociales encaja a la perfección con la forma de funcionamiento de las universidades, es intrínseco a su naturaleza (misión y visión), ya en este tipo de instituciones públicas el diálogo con la sociedad juega un papel crucial, sobre todo para el desarrollo de las nuevas misiones de la universidad.

Desde la perspectiva de las universidades, sería preciso avanzar en sus estrategias de diferenciación, consolidar una mentalidad emprendedora que impulse la tercera misión de la universidad, construir sistemáticamente la imagen y la reputación de la universidad para, entre otros fines, beneficiarse de programas de merchandising (o comercialización de distintos productos y servicios asociados a las mismas, como -por ejemplo- ya viene haciendo en cierto modo la Universidad de Salamanca) y de fundraising (Cambridge, Oxford, Tecnológico de Zurich, etc.). En este sentido, la relación con los antiguos alumnos adquiere una importancia capital (como muestra 
en nuestro país el ejemplo de algunas universidades privadas, como la de Navarra).

En suma, la captación de fondos privados por parte de las universidades públicas españolas requiere de importantes cambios culturales de difícil verificación en el corto plazo; pero que habría que poner en marcha de inmediato si se quieren obtener resultados aceptables en un futuro cercano. Por otro lado, también es necesario fomentar en España una "cultura de la filantropía" para vincular debidamente a los potenciales donantes con los objetivos, función y misiones de la universidad, lo que exigiría un esfuerzo sociopolítico adicional. Además habría que difundir entre las universidades públicas españolas la "cultura de pedir", para lo cual sería necesario el establecimiento de estructuras organizativas profesionales que extendieran la misma en el interior de la institución y establecieran procesos eficientes para dirigirse a los posibles donantes. Habría que concienciar a la sociedad de que es necesario un esfuerzo en investigación y que hay que apoyarlo con recursos no sólo públicos, sino también privados, y para ello, es necesario mejorar el tratamiento fiscal y los convenios de colaboración universidad-empresa y realizar buenas campañas de marketing para acercar y difundir los objetivos de la universidad a la sociedad.

\section{Referencias bibliográficas}

AECA (2006) La Semántica de la Responsabilidad Social Corporativa, Documento n. ${ }^{\circ}$ 3; Madrid: AECA.

AECA (2007) Gobierno y Reponsabilidad Social de la empresa; Madrid: AECA.

Bueno, E. y Casani, F. (2007) "La tercera misión de la universidad. Enfoques e indicadores básicos para su evolución". Economía Industrial, n. 366, pp. 43-59.

Casani, F. (2007) Los nuevos desafíos para el gobierno de la universidad. Madrid, revista de Investigación en gestión de la innovación y la tecnología, n. ${ }^{\circ} 41$. Obtenido de: http://www.madrimasd.org/revista/revista4l/tribuna/ tribuna3.asp

Casani, F, y Pérez-Esparrells, C. (2009) "La responsabilidad social en las universidades públicas españolas: vectores de cambio en la gobernanza", Investigaciones en Economía de la Educación, n. ${ }^{\circ}$ 4, pp.127-137. 
Casani, F. Rodríguez Pomeda, J. y Martín, J.I. (2007) "Los retos para el gobierno y la gestión de la universidad: la dirección estratégica y el enfoque stakeholder"; XIV Congreso de la AECA (mimeo).

Clark, B. (1998) Creating Entrepreneurial Universities: Organizational Pathways of Transformation; New York, NY: International Association of Universities and Elsevier Science.

Comisión de Financiación (2007) Financiación del sistema universitario español. Madrid: Ministerio de Educación y Ciencia, Consejo de Coordinación Universitaria. Obtenido de: http://www.educacion.es/dctm/ mepsyd/educacion/universidades/educacion-superior-universitaria/ financiacion/financiacion.pdf?documentId=0901e72b8004879d

Comisión Mixta de Financiación Universitaria (2010) Documento de reflexión sobre la mejora de las politicas de financiación de las universidades para promover la excelencia. Madrid: Ministerio de Educación, Consejo de Universidades. Obtenido de: http://educacion.es/dctm/ministerio/ horizontales/documentacion/enero/documento-financiacion 1 . pdf?documentId=0901e72b800adffa

Department for Education and Skills (2004) Increasing Voluntary Giving to Higher Education. Task Force Report to Government. Obtenido de: http:// www.dfes.gov.uk/hegateway/uploads/Increasing $\% 20$ Voluntary $\% 20$ Giving\%20to\%20Higher\%20Education\%20\%20Task\%20Force\%20 Report\%20to\%20Government.pdf

European Commission (2003) The role of the universities in the Europe of knowledge; COM (2003); 58 final; Brussels, 02.05.2003.

European Commission (2005) Mobilising the brainpower of Europe: enabling universities to make their full contribution to the Lisbon Strategy, COM (2005) 152 final; Brussels, 20.04.2005.

European Commission (2006) Delivering on the modernisation agenda for universities: education, research and innovation, COM (2006), Brussels, 10.05.2006.

European Commission-Directorate General for Research (2008) Engaging Philanthropy for University Research. Luxembourg: Office for Official Publications of the European Communities. Obtenido de: http:// ec.europa.eu/invest-in-research/pdf/download_en/rapport2007_final. pdf

Gilby, N.; Lloyd, C., y Finch, S. (2009) Ross-CASE Survey 2007-8. Final Report. Obtenido de: http://www.rosscasesurvey.org.uk

Global Reporting Initiative, Gri (2006) Sustainability Reporting Guidelines; Amsterdam: GRI. 
Jongbloed, B.; Enders, J. y Salerno, C. (2008) "Higher Education and its communities: interconnections, interdependencies and a research agenda"; Higher Education 56, pp. 303-324.

Kaplan, A.E. (2009) Voluntary Support of Education. New York, NY: Council for Aid to Education (CAE).

Leslie, L., y Ramey, G. (1988) Donor Behavior and Voluntary Support for Higher Education Institutions; Journal of Higher Education, 59, pp. $117-32$.

Lozano, J.M. (2001) La empresa ciudadana: Un reto de innovación. ESADE.

Lozano, J.M. (2007) "Promoción pública de la responsabilidad social empresarial"; Ekonomiaz, n. ${ }^{\circ}$ 65, pp. 108-127.

Mitchell, R.K.; Agle, B.R. y Wood, D.L. (1997) "Toward a Theory of Stakeholder Identification and Salience: Defining the Principle of Who and What Really Counts"; Academy of Management Review, 22(4), pp. 853-886.

Mora, J.-G., y Nugent, M. (1998) "Seeking new resources for European universities: the example of fundraising in the U.S."; European Journal of Education, 33(1), pp. 113-129.

Neave, G. (2000) The Universities' responsibilities to society; Oxford: Pergamon.

Pérez-Esparrells, C. (2004) La educación universitaria en España: el vínculo entre financiación y calidad. Revista de Educación, n. ${ }^{\circ} 335$, pp. 305316.

Porter, M.E. y Kramer, M.R. (2006) The Competitive Advantage of Corporate Philantrophy, Boston, MA: HBS Press.

Shattock, M. (2003) Managing Successful Universities, Buckingham: The Society for Research in Higher Education and Open University Press.

Scott, G. (2003) "Effective Change Management in Higher Education"; Educase Review. Volume 38, number 6, November/December 2003.

Recibido: 12/09/2010

Aceptado: 10/11/2010 